\title{
Opções terapêuticas e perspectivas no tratamento da alopecia androgenética
}

\author{
Therapeutic options and perspectives in the treatment of androgenetic alopecia
}

Opciones y perspectivas terapéuticas en el tratamiento de la alopecia androgenética

Rafaela Lepkoski Chaves ${ }^{1 *}$, Diego Martins Sanson², Eduarda Faria do Nascimento ${ }^{3}$, Julia Cristina de Oliveira ${ }^{4}$, Júlia Peres Esteves ${ }^{5}$, Kellen Luanny Silva ${ }^{2}$, Marina Nascimento Gomes ${ }^{6}$, Rafaella Bosi Castro de Oliveira ${ }^{7}$, Renata Viana Hoffmann Monteiro Guedes ${ }^{8}$, Milena Ferreira Gandra ${ }^{9}$.

\section{RESUMO}

Objetivo: Apresentar e discutir as principais formas terapêuticas para a alopecia androgenética (AAG), em ambos os sexos. Revisão bibliográfica: $A$ partir da revisão realizada verificou-se que a AAG é uma dermatopatologia multifatorial, que acomete ambos os sexos. Entre os tratamentos propostos, a Finasterida se mostrou mais eficaz em homens do que em mulheres; a Dutasterida, por sua vez, tem sido utilizada em casos que não obtenham resultados com a Finasterida, podendo ainda apresentar maior aumento da quantidade de fios do que o primeiro; o Minoxidil, na sua forma tópica, é a primeira opção em casos de alopecia moderada; e o Tratamento Cirúrgico, que se dá por cirurgia de redução do couro cabeludo, transplante capilar, ou a combinação de ambos. Há ainda algumas terapêuticas que são menos utilizadas em menor frequência ou que ainda estão em estudo como os Análogos e Antagonistas de Prostaglandinas, a Terapia a Laser e a Terapia com Células Tronco. Considerações finais: Observou-se que os estudos são realizados mais frequentemente em homens, visto que são os mais acometidos. Além disso, notou-se a necessidade de pesquisas sobre alguns medicamentos e sobre a associação entre certos tratamentos, assim como algumas vantagens entre eles.

Palavras-chave: Dermatologia, Terapêutica, Alopecia.

\begin{abstract}
Objective: To present and discuss the main therapeutic forms for androgenetic alopecia (AGA) in both sexes. Literature review: The review showed that AGA is a multifactorial dermatopathology that affects both sexes. Among the treatments proposed, Finasteride has shown to be more effective in men than in women; Dutasteride, on the other hand, has been used in cases that do not get results with Finasteride, and may even present a greater increase in the amount of hair strands than the former; Minoxidil, in its topical form, is the first optionin cases of moderate alopecia; and the Surgical Treatment, which may be by scalp reduction surgery, hair transplantation, or a combination of both. There are also some therapies that are less frequently used or that are still being studied, such as Prostaglandin Analogues and Antagonists, Laser Therapy, and Stem Cell Therapy. Final considerations: It was observed that the studies are carried out more frequently in men, since they are the most affected. Moreover, it was noted the need for research on some drugs and on the association between certain treatments, as well as some advantages among them.
\end{abstract}

Key words: Dermatology, Therapeutics, Alopecia.

\footnotetext{
1 Universidade de Caxias do Sul (UCS), Caxias do Sul - RS. *E-mail: rafalepkoski@gmail.com

2 Universidade Federal de Juiz de Fora (UFJF), Juiz de Fora - MG.

3 Universidade Luterana do Brasil (ULBRA), Canoas - RS.

${ }^{4}$ Universidade Federal de Ouro Preto (UFOP), Ouro Preto - MG.

${ }^{5}$ Pontifícia Universidade Católica de Campinas (PUC), Campinas - SP.

${ }^{6}$ Faculdade Ciências Médicas de Minas Gerais (FCMMG), Belo Horizonte - MG.

${ }^{7}$ Instituto Metropolitano de Ensino Superior (IMES/ Univaço), Ipatinga - MG.

${ }^{8}$ Faculdade de Medicina de Barbacena (FAME/FUNJOB), Barbacena - MG.

${ }^{9}$ Centro Universitário de Caratinga (UNEC), Caratinga - MG.
} 


\section{RESUMEN}

Objetivo: Presentar y discutir las principales formas terapéuticas para la alopecia androgenética (AGA) em ambos sexos. Revisión de la literatura: La revisión mostró que la AGA es uma dermatopatología multifactorial que afecta a ambos sexos. Entre los tratamientos propuestos, el Finasteride há demostrado ser más eficaz em los hombres que em las mujeres; el Dutasteride, por su parte, se há utilizado em los casos que no obtienen resultados com el Finasteride, y además puede presentar um mayor aumento de la cantidad de cabellos que el primero; el Minoxidil, em su forma tópica, es la primera opción em los casos de alopecia moderada; y el Tratamiento Quirúrgico, que puede ser mediante cirugía de reducción del cuero cabelludo, trasplante capilar, o uma combinación de ambos. También hay algunas terapias que se utilizan com menos frecuencia o que aún están em estudio, como los análogos y antagonistas de las prostaglandinas, la terapia com láser y la terapia com células madre. Consideraciones finales: Se observó que los estudios se realizan com mayor frecuencia em hombres, ya que son los más afectados. Además, se señaló la necesidad de investigar sobre algunos medicamentos y sobre la asociación entre ciertos tratamientos, así como algunas ventajas entre ellos.

Palabras clave: Dermatología, Terapéutica, Alopecia.

\section{INTRODUÇÃO}

A Alopecia Androgenética (AAG) é uma dermatopatologia multifatorial poligeneticamente determinada, que acomete ambos os sexos e inicia-se na adolescência, quando o estímulo hormonal androgênico favorece que, em cada ciclo capilar, os fios venham progressivamente mais finos e menos pigmentados, em virtude da miniaturização progressiva não cicatricial do folículo piloso pelo encurtamento da fase anágena (KELLY Y, et al., 2016).

A prevalência das queixas ambulatoriais de AAG na população é relativamente alta. Na etnia caucasiana, $80 \%$ do sexo masculino é acometido, de forma que a idade compreendida entre os 18 aos 29 anos corresponde a $12 \%$ dos casos, e entre 40 a 49 anos chega a aproximadamente $50 \%$. No sexo feminino, atinge cerca de $40 \%$ a $50 \%$ da população acima dos 45 anos e o afinamento capilar começa entre 12 e 40 anos, apresentando incidência igual ao sexo masculino. Na etnia africana, 14,6\% dos homens possuem AAG em contrapartida de $3,5 \%$ das mulheres da população em geral. De forma semelhante, $14,1 \%$ dos homens asiáticos são acometidos pela patologia (ROSSI A, et al., 2016; KANTI V, et al., 2018).

Além disso, as manifestações da AAG se associam a uma diferença de padrão distributivo em homens e mulheres predispostos. No sexo feminino, a região central do escalpo é mais acometida e há a possibilidade de associação com irregularidade dos ciclos menstruais, acne, obesidade, hirsutismo e síndrome metabólica. Nos homens, as áreas mais afetadas são a região frontal bilateral e coronal. Embora o nome da doença esteja relacionado ao hormônio masculino, grande parte das análises sanguíneas laboratoriais evidenciam níveis hormonais normais (VAROTHAI S e BERGFELD WF, 2014).

As queixas de queda de cabelo na prática dermatológica são frequentes e apresentam três principais diagnósticos diferenciais: alopecia androgenética, alopecia areata e eflúvio telógeno. Desse modo, é de grande significância a realização de uma anamnese completa, assim como exame físico e dermatoscópico, para condução e instituição de terapêutica adequada (VAROTHAI S e BERGFELD WF, 2014). Segundo Gupta S, et al. (2019), embora seja uma condição fisiológica, a AAG acarreta prejuízos na qualidade de vida dos seus portadores, visto que a perda de cabelo pode levar a uma imagem distorcida de si mesmo, o que impacta negativamente na saúde mental, na autoestima e na vida social, dos portadores dessa condição.

Atualmente, a terapêutica instituída para o tratamento da AAG visa retardar a progressão da queda capilar e aumentar a cobertura do couro cabeludo dos pacientes, interrompendo o processo de miniaturização do fio e melhorando a densidade capilar. A cada ano, novas terapias são introduzidas ao escopo clínico e as mais citadas no tratamento clínico são a finasterida, dutasterida, minoxidil oral e tópico, antagonistas e agonistas de prostaglandinas, plasma rico em plaquetas, terapia a laser e com células-tronco (MULINARI-BRENNER F, et al., 2011; VAÑÓ-GALVAN S e CAMACHO F, 2017; HESSELER M J e SHYAM NL, 2019). 
Existem, ainda, opções de tratamentos cirúrgicos, baseados na redução do couro cabeludo e/ou no transplante capilar. Estes tratamentos são considerados quando o paciente apresenta perda capilar excessiva, bem como quando os resultados foram refratários ao tratamento farmacológico (ROSSI A, et al., 2016; VAROTHAI S E BERGFELD WF, 2014). Diante desse contexto, o presente estudo tem como objetivo descrever e discutir as atuais opções terapêuticas no tratamento da alopecia androgenética, em ambos os sexos, a partir de uma revisão da produção científica atual sobre o tema.

\section{REVISÃO BIBLIOGRÁFICA}

A AAG é uma doença hereditária, dependente de hormônios e caracterizada por alteração no ciclo capilar, que leva, progressivamente, à minimização do folículo capilar culminando em fios terminais mais finos, curtos e menos pigmentados. Sua prevalência não está totalmente estabelecida, mas sabe-se o que o seu aparecimento está relacionado com idade, sexo e etnia. Na etnia caucasiana, cerca de $80 \%$ dos homens são acometidos pela AAG e, no sexo feminino, o acometimento é de cerca de $40 \%$ a $50 \%$ nas mulheres acima dos 45 anos. A condição é menos prevalente em negros e asiáticos (ROSSI A, et al., 2016).

Fisiologicamente, o folículo piloso passa por três estágios de desenvolvimento: o proliferativo (fase anágena), o involutivo (fase catágena) e o de repouso (fase telógena). No couro cabeludo normal, a fase anágena possui duração de dois a sete anos, a catágena de duas semanas e a telógena de aproximadamente três meses. Porém, já foram demonstradas fases de atraso entre a queda e a reposição de uma nova haste, que é denominada fase quenógena, em que o folículo se encontra vazio e é uma fase de fundamental importância para a compreensão da fisiopatogenia da AAG (ROUSSO DE e KIM SW, 2014).

Nessa doença, além de haver um aumento do número de folículos pilosos em repouso na fase quenógena, também ocorre um término prematuro da fase anágena pela redução da expressão de fatores estimulantes e aumento das citocinas que promovem apoptose (ROUSSO DE e KIM SW, 2014). A queixa mais frequentemente relatada na $A A G$ é a do afinamento dos fios. Os cabelos ficam mais ralos e, progressivamente, o couro cabeludo mais aberto (KANTI V, et al., 2018).

Entretanto, a região de acometimento costuma diferir entre homens e mulheres e a progressão da doença é variável, sendo mais exacerbada quanto mais cedo for o início. A AAG masculina costuma iniciar após a puberdade com recessão bitemporal simétrica, evoluindo com acometimento do vértex. O padrão feminino, por sua vez, costuma se apresentar significativamente pior após a menopausa e é caracterizado por afinamento difuso dos cabelos, poupando a linha de implantação frontal, com rarefação na região frontoparietal e no vértex (VAROTHAI S e BERGFELD WF, 2014).

O tratamento da AAG visa aumentar a cobertura do couro cabeludo e também retardar a progressão da queda capilar e pode ser realizado de maneira tópica, sistêmica e/ou cirúrgica, porém, as medidas farmacológicas diferem entre os homens e as mulheres. Além disso, medidas complementares podem ser adotadas como a exclusão do uso de medicações que podem causar eflúvio telógeno, estímulo a uma dieta balanceada, com níveis adequados de proteína e ferro, além do tratamento de outras desordens do couro cabeludo como a dermatite seborreica. Além disso, em pacientes obesos, recomenda-se o controle do peso de forma a contribuir com a melhora do perfil hormonal como um todo (MULINARI-BRENNER F, et al., 2011).

Em relação aos tratamentos propostos, suas respectivas indicações e aplicabilidades clínicas serão discutidos individualmente.

\section{Minoxidil}

O minoxidil tem sido uma das principais terapêuticas utilizadas no tratamento da AAG, sendo considerado primeira opção de tratamento tópico em homens e mulheres com quadro moderado de alopecia. Este medicamento foi originalmente desenvolvido em meados dos anos 70 para tratamento da hipertensão arterial sistêmica, contudo, a hipertricose foi notada repetidamente como um efeito colateral nos usuários da droga. O real mecanismo de ação da droga na AAG ainda não está completamente esclarecido, mas acredita-se que o efeito no crescimento capilar tem grande relação com a abertura de canais de potássio e seu consequente 
aumento do fluxo sanguíneo cutâneo e dos níveis de fator de crescimento endotelial (ROSSI A, et al., 2012; KELLY Y, et al., 2016).

Atualmente, indica-se o tratamento tópico com minoxidil na concentração de $5 \%$ para homens e 2 a $5 \%$ para mulheres. Contudo, são relatados maiores efeitos colaterais como prurido e hipertricose em locais não desejados quando utilizada a concentração de 5\% (KANTI V, et al., 2018).

Estudos demonstram que o pico de crescimento capilar ocorre em torno de 24 semanas após o início do tratamento e que o uso contínuo ajuda a manter seus benefícios e desacelera a perda capilar. Entretanto, deve ser esclarecido aos pacientes que a interrupção do uso diário resulta em perda do efeito em 4-6 meses (VAROTHAI S E BERGFELD WF, 2014).

O uso oral de minoxidil, por sua vez, tem-se mostrado controverso devido aos possíveis efeitos colaterais da droga quando em concentrações de 10-40 mg. Dentre tais efeitos adversos podemos citar a retenção de sódio e água que pode culminar em edema, ganho de peso ou até mesmo congestão pulmonar em pacientes nefropatas. Contudo, estudos recentes mostraram que quando utilizado em concentrações inferiores a $5 \mathrm{mg}$, o minoxidil teve considerável aumento da tolerabilidade à droga e diminuição dos efeitos colaterais (RANDOLPH M e TOSTI A, 2021).

\section{Finasterida}

A finasterida é uma droga que está disponível desde 1990, e é classificada como uma inibidora da 5aRedutase do tipo 2. Ela age diminuindo dois terços da conversão de testosterona em di-hidrotestosterona (DHT), principal responsável pela miniaturização dos folículos na AAG. Essa droga tem uma eficácia já comprovada para tratamento da AAG masculina, e é eficaz principalmente para pacientes jovens ou com acometimento da região do vertex, na dose de $1 \mathrm{mg} /$ dia. Observa-se um aumento dos fios em $80 \%$ dos casos tratados com a finasterida na dose proposta, tornando-se mais numerosos nos primeiros 12 meses e mais espessos após este período (MULINARI-BRENNER F, et al., 2011).

Orienta-se que, para manutenção destes benefícios, a finasterida deve ser mantida durante toda a vida, uma vez que seus efeitos terminam após 12 meses da interrupção do uso. Em relação às mulheres, a finasterida mostrou-se pouco eficaz na maioria dos casos (MULINARI-BRENNER F, et al., 2011).

Mesmo não sendo hepatotóxica ou nefrotóxica, a finasterida é contraindicada em pacientes com distúrbios hepáticos devido à sua metabolização no fígado. É contraindicado também a doação de sangue por pacientes em uso deste medicamento, de forma a evitar que ocorra a transferência da droga a gestantes, evitando efeitos teratogênicos (BELKNAP SM, et al., 2015).

Em relação aos efeitos adversos, cerca de $2 \%$ dos pacientes relatam um ou mais efeitos como a queda da libido, disfunção erétil e diminuição do volume ejaculatório, entretanto, tais ocorrências não tornam obrigatória a interrupção do tratamento. Em alguns pacientes em tratamento com $1 \mathrm{mg} / \mathrm{dia}$ de finasterida, podem ocorrer efeitos adversos sexuais permanentes e, mesmo a ocorrência sendo inferior a $1 \%$ e com poucas evidências em relação a isso, é necessário informar a possibilidade deste risco ao paciente. Outros efeitos colaterais relatados, que podem estar relacionados ao uso da finasterida são a depressão, ansiedade, ginecomastia uni ou bilateral e mudança dos parâmetros e da qualidade do sêmen (BELKNAP SM, et al., 2015).

\section{Dutasterida}

A dutasterida é uma inibidora da $5 \alpha$-Redutase do tipo 1 e 2, e também age na redução da quantidade de DHT de forma mais eficaz que a finasterida, diminuindo em até $90 \%$ os níveis de DHT. Além disso, a dutasterida é uma boa alternativa para os casos em que a finasterida mostrou-se pouco eficaz e é capaz de gerar um aumento da quantidade de fios maior que a finasterida (ROUSSO DE e KIM SW, 2014; ZHOU Z, et al., 2019). A dose recomendada é de $0,5 \mathrm{mg} / \mathrm{dia}$ e é relatado, como efeitos adversos, disfunções sexuais, diminuição da libido e a dificuldade de ejaculação, porém em menor prevalência quando comparada à finasterida (LEE S, et al., 2019). 


\section{Análogos e antagonistas de prostaglandinas}

O papel dos análogos da prostaglandina no tratamento da alopecia foi percebido pelo alongamento e pigmentação dos cílios e sobrancelhas ocorridos como um efeito colateral em pacientes que usam o análogo de prostaglandina F2 (PGF2) tópico Latanoprosta para o tratamento de glaucoma. Em 2008, o FDA aprovou o Bimatoprost, outro análogo de PGF2, para o tratamento de hipotricose de cílios (VAROTHAI S E BERGFELD WF, 2014; VACCARO M, et al., 2015).

Estudos mostraram que, homens usando Latanoprosta na concentração de $0,1 \%$, uma vez ao dia em uma pequena área do couro cabeludo por 24 semanas, tiveram aumento significativo na densidade do cabelo em comparação com a linha de base e o placebo, sem efeitos colaterais, exceto uma reação eritematosa localizada (KELLY Y, et al., 2016). Estudos recentes revelam, ainda, que um aumento dos níveis de prostaglandina D2 (PGD2) está relacionado com a miniaturização de folículos capilares e, além disso, aplicações tópicas de PGD2 também inibiu o crescimento do cabelo. Em contraste, PGF2 e PGE2 são sinérgicos, o resultado do qual induz o crescimento do cabelo e prolonga o anágeno (VAROTHAI $S$ e BERGFELD WF, 2014).

\section{Plasma rico em plaquetas}

O plasma rico em plaquetas (PRP) é um tipo de tratamento que ganhou popularidade para AAG por ser autólogo, ter baixa invasividade, ausência de efeitos colaterais importantes e custo mais acessível em comparação com a cirurgia de restauração capilar. O mecanismo de ação do PRP baseia-se na ativação das plaquetas, com consequente liberação de numerosos fatores de crescimento e citocinas como parte do processo de cicatrização de feridas. Dessa forma, quando injetadas no couro cabeludo, as plaquetas no PRP tornam-se ativadas e liberam múltiplos fatores de crescimento que participam da ativação do fibroblasto, síntese de colágeno, estimulação da matriz extracelular e superexpressão de fatores de crescimento endógenos, promovendo, assim, o crescimento do cabelo (STEVENS J, et al., 2019; HESSELER MJ e SHYAM NL, 2019).

O PRP tem uma base científica teórica para apoiar seu uso em cirurgia de restauração capilar e tratamento clínico da AAG. No entanto, ainda não há nenhuma informação baseada em evidências sobre protocolo de administração e não existem dados sobre a dose e frequência ideais de injeções. Dessa forma, há a necessidade de ensaios clínicos randomizados e controlados por placebo envolvendo um grande número de pacientes para estabelecer a real eficácia e duração dos resultados do PRP (MONTERO ME, et al., 2015).

\section{Terapia com células tronco}

O tratamento com células tronco visa repor essas células perdidas e consequente ativação do folículo capilar, visto que a patogênese da AAG envolve uma resposta imune que culmina na destruição das células tronco foliculares do folículo piloso, levando a perda de cabelo irreversível. Os estudos indicam uma maior predileção pelo tecido adiposo para coletar as células tronco adultas, uma vez que sua técnica de obtenção é menos invasiva e tem-se a possibilidade de encontrar multi-linhagens. (GENTILI P e GARCOVICH S, 2019; WOLFF H, et al., 2016).

Os adipócitos intradérmicos secretam hormônios, fatores de crescimento e citocinas, modulando respostas imunológicas e celulares. No tratamento com células tronco observou-se aumento significativo na contagem de cabelos pelo aumento da capacidade de desenvolvimento dos folículos. As células tronco podem ser aplicadas via intracutânea e também por microagulhamento diretamente no couro cabeludo por duas semanas, mostrando resultados positivos no que diz respeito ao aumento da densidade e espessura do cabelo (KELLY Y, et al., 2016; ROSSI A, et al., 2016).

\section{Terapia a laser}

O mecanismo de ação desta terapia está associado à produção transitória de espécies reativas de oxigênio induzidas pelo laser que ativam vias de sinalização celular que ativam o folículo piloso, acelerando o crescimento do cabelo. Além disso, o laser aumenta o fluxo sanguíneo local, aumenta o número de mitoses celulares, estimula células tronco do folículo capilar e aumenta o metabolismo local favorecendo a produção 
de trifosfato, e atividade celular com efeitos anti-inflamatórios, gerando efeito protetor (VAÑÓ-GALVAN S e CAMACHO F, 2017; DONOVAN J, et al., 2021).

O tratamento a laser mostrou aumento na contagem de cabelos quando usado entre 16 e 26 semanas, tanto em homens como em mulheres com AAG. Foi evidenciado que o tratamento com frequência de três vezes por semana mostrou melhorias na densidade do cabelo e maior resistência à tração. O comprimento de onda entre 650 e 900 nm é evidenciado como uma opção eficaz (KANTI V, et al., 2018; ROSSI A, et al., 2016).

\section{Tratamento cirúrgico}

O tratamento cirúrgico deve ser considerado nos casos em que o paciente apresenta perda capilar excessiva, assim como nos pacientes em que os resultados foram refratários ao tratamento farmacológico. Em geral, os pacientes que mais se beneficiam são aqueles que possuem mais de 25 anos e queda de cabelo estabilizada. As opções cirúrgicas incluem a cirurgia de redução do couro cabeludo, transplante capilar, ou a combinação de ambos (VAROTHAI S e BERGFELD WF, 2014).

O transplante capilar é a opção cirúrgica de preferência pois, com os avanços desse tipo de técnica nos últimos anos, é considerado um procedimento microcirúrgico e menos agressivo, por reduzir o trauma e manter a integridade do escalpo. Existem vários métodos para a realização do transplante capilar, e a indicação para cada um depende da área de alopecia do paciente e também de qual é o resultado estético desejado. Porém, é unânime em que todas as técnicas os folículos sejam retirados de uma área saudável e sejam transferidos para a área em que há rarefação capilar, consistindo, esse princípio, na dominância do folículo doador, uma vez que os folículos capilares localizados nas áreas insensíveis aos andrógenos possuem suas propriedades preservadas mesmo quando transplantados para um couro cabeludo andrógeno dependente (KELLY Y, et al., 2016).

O objetivo dessa terapêutica é que, com o tempo, o folículo transplantado se adapte em sua nova localização, fazendo com que, a partir de então, o crescimento capilar aconteça de forma natural. Assim, o resultado inicial costuma ser insatisfatório, uma vez que só se torna evidente de seis a oito meses após a realização do procedimento. Por isso, é indicado o uso de terapia farmacológica adjuvante, sendo o Minoxidil tópico bem indicado. Uma limitação dessa opção terapêutica é não ter eficácia na progressão da AAG, ou seja, não impede que a doença continue progredindo (ROUSSO DE e KIM SW, 2014).

\section{CONSIDERAÇÕES FINAIS}

A partir da análise bibliográfica, percebe-se que há variadas modalidades terapêuticas para a alopecia androgenética e isso demonstra a importância de se realizar a avaliação específica e indicação de tratamento para cada caso. Além disso, nota-se a existência de mais opções de tratamento para homens por serem o grupo mais afetado por esse quadro. Ademais, é necessária a realização de mais pesquisas para novas opções de tratamento como a utilização do PRP e antagonistas de prostaglandinas, bem como para analisar a associação de diferentes métodos de tratamentos. Em alguns casos podem ser observados, ainda, tratamentos com algumas vantagens em relação a outros, como o uso da dutasterida em substituição a finasterida e a PRP no lugar da cirurgia de restauração capilar.

\section{REFERÊNCIAS}

1. BELKNAP SM, et al. Adverse event reporting in clinical trials of finasteride for androgenic alopecia: a meta-analysis. JAMA dermatology, 2015;151(6): 600-606.

2. DONOVAN J, et al. UpToDate - Treatment of androgenetic alopecia in men. 2021. Disponível em: https://www.uptodate.com/contents/treatment-of-androgenetic-alopecia-inmen. Acessado em: 30 de março de 2021.

3. GENTILE P, GARCOVICH S. Advances in regenerative stem cell therapy in androgenic alopecia and hair loss: Wnt pathway, growth-factor, and mesenchymal stem cell signaling impact analysis on cell growth and hair follicle development. Cells, 2019; 8(5): 466.

4. GUPTA S, et al. Quality of life assessment in patients with androgenetic alopecia.International Journal of Trichology, 2019;11: 147-152. 
5. HESSELER MJ, SHYAM NIKHIL. Platelet-Rich Plasma and Its Utilities in alopecia: A Systematic Review. Wolters Kluwer Health, 2020; 46(1): 93-102.

6. KANTI V, et al. Evidence-based (S3) guideline for the treatment of androgenetic alopecia in women and in men - short version. Journal of the European Academy of Dermatology and Venereology, 2018; 32(1): 11-22.

7. KELLY Y, et al. Androgenetic alopecia: an Update of Treatment Options. Drugs. 2016; 76(14):1349-1364.

8. LEE S, et al. Adverse Sexual Effects of Treatment with Finasteride or Dutasteride for Male Androgenetic alopecia: A Systematic Review and Meta-analysis. Acta Dermato-Venereologica, 2019; 99(1):12-17.

9. MONTERO ME, et al. Platelet-rich plasma: applications in dermatology. Actas Dermo-Sifiliográficas, 2015;106(2): 104-11.

10. MULINARI-BRENNER F, et al. Entendendo a alopecia androgenética. Surgical \& Cosmetic Dermatology, 2011; 3(4):329-337.

11. RANDOLPH M, TOSTI A. Oral minoxidil treatment for hair loss: A review of efficacy and safety. Journal of the American Academy of Dermatology, 2021;84(3):737-746.

12. ROSSI A, et al. Minoxidil Use in Dermatology, Side Effects and Recent Patents. Recent Patents on Inflammation \& Allergy Drug Discovery, 2012; 6:130-136.

13. ROSSI A, et al. Multi-therapies in androgenetic alopecia: review and clinical experiences. Dermatologic Therapy, 2016; 29(6):424-432.

14. ROUSSO DE, KIM SW. A review of medical and surgical treatment options for androgenetic alopecia. JAMA Facial Plastic Surgery, 2014;16(6):444-450.

15. STEVENS J, et al. Platelet-rich plasma for androgenetic alopecia: A review of the literature and proposed treatment protocol. International Journal of Women's Dermatology, 2019; 5:46-53.

16. VACCARO M, et al. Erosive pustular dermatosis of the scalp following topical latanoprost for androgenetic alopecia. Dermatologic Therapy, 2015;28(2):65-67.

17. VAÑÓ-GALVAN S, CAMACHO F. New treatments for hair loss. Actas Dermo-Sifiliográficas, 2017; $108(3): 221-228$.

18. VAROTHAI S, BERGFELD WF. Androgenetic alopecia: An Evidence-Based Treatment Update. American journal of clinical dermatology, 2014; 15(3):217-230.

19. WOLFF H, et al. The diagnosis and treatment of hair and scalp diseases. Deutsches Ärzteblatt International, 2016; 113(21):377.

20. ZHOU Z, et al. The efficacy and safety of dutasteride compared with finasteride in treating men with androgenetic alopecia: a systematic review and meta-analysis. Clinical Interventions in Aging, 2019; 14:399-406. 